\title{
PRENATAL DIAGNOSIS
}

METABOLIC FINGERPRINTING OF CHORIONIC VILLOUS SAMPLES IN NORMAL PREGNANCY AND CHROMOSOMAL DISORDERS

\begin{tabular}{|c|c|}
\hline Journal: & Prenatal Diagnosis \\
\hline Manuscript ID & PD-19-0093.R1 \\
\hline Wiley - Manuscript type: & Original Article \\
\hline $\begin{array}{l}\text { Date Submitted by the } \\
\text { Author: }\end{array}$ & 28-Mar-2019 \\
\hline Complete List of Authors: & $\begin{array}{l}\text { Murgia, Federica; University of Cagliari, Biomedical sciences } \\
\text { Iuculano, Ambra; Ospedale Microcitemico, Prenatal Diagnosis } \\
\text { peddes, cristina; Azienda Ospedaliera Brotzu, Prenatal Diagnosis } \\
\text { Santoru, Maria Laura; University of Cagliari, Biomedical sciences } \\
\text { Tronci, Laura; University of Cagliari, Biomedical sciences } \\
\text { Deiana, Monica; University of Cagliari, Biomedical sciences } \\
\text { Atzori, Luigi; University of Cagliari, Biomedical sciences } \\
\text { Monni, Giovanni; Ospedale Microcitemico, Prenatal Diagnosis }\end{array}$ \\
\hline KeyWords: & $\begin{array}{l}\text { Placental development, Chorionic villous, Trisomies, Metabolomics, Polyol } \\
\text { pathway, Oxidative stress }\end{array}$ \\
\hline
\end{tabular}

\section{SCHOLARONE" Manuscripts}




\section{METABOLIC FINGERPRINTING OF CHORIONIC VILLOUS SAMPLES IN NORMAL \\ 2 PREGNANCY AND CHROMOSOMAL DISORDERS}

3 Federica MURGIA $^{1 *}$, Ambra IUCULANO ${ }^{2 *}$, Cristina PEDDES $^{2}$, Maria Laura SANTORU ${ }^{1}$,

4 Laura TRONCI ${ }^{1}$, Monica DEIANA ${ }^{1}$, Luigi ATZORI ${ }^{1 * *}$, Giovanni MONNI ${ }^{2 * *}$.

$5 \quad *$ Equally contributing Authors

$6 \quad * *$ Equally contributing Authors

7

81 1. Department of Biomedical Sciences, Clinical Metabolomics Unit, University of Cagliari.

9 102

11 Pediatrico Microcitemico A.Cao, Cagliari, Italy

12

13

14

15

16

17

18 4 45 46 47 $48 \quad 21$ 49 50 51 52 53 54 55 56

Correspondence:

(e-mail: prenatalmonni@tiscali.it)

Manuscript word: 36863991; Table: 2; Figure-: 5

2. Department of Prenatal and Preimplantation Genetic Diagnosis and Fetal Therapy, Ospedale

Dr Giovanni Monni, Department of Prenatal and Preimplantation Genetic Diagnosis and Fetal Therapy, Ospedale Microcitemico, Via E. Jenner n/n, 09121 Cagliari, Italy 19

Short running title: Metabolomic study of Chorionic villous samples 


\section{BULLETED STATEMENTS}

- Currently, there are few studies that evidence specific changes of the placental metabolic profile during the first trimester of pregnancy.

- This study analyzes alterations in placental metabolites composition in euploid and aneuploid cases. Polyols might have a crucial role in energy production. In aneuploid cases the exceeding activation of polyol pathway leads to the increase of oxidative stress. Myoinositol and cholesterol decrease in cases of aneuploidy.

\section{Bulleted statement}

- Switch from anabolic to catabolic placental metabolism during the first trimester is a typical feature of pregnancy to promote fetal growth, but only few scientific studies evidenced specific changes of the placental metabolic profile during this pregnant period. Changes in maternal metabolic composition, has been observed by the analysis of biofluids and similar changes could be observed also in placenta tissue suggesting biological mechanisms in normal and pathological pregnancies

- This study allowed to better understand alterations in placental metabolites composition in euploid and aneuploid cases. Key findings of this study evidenced that in normal pregnancy polyols might have a crucial role in energy production. In aneuploid cases the exceeding activation of polyol pathway leads the increase of oxidative stress. Moreover, myo-inositol and cholesterol were found decresed in cases of aneuploidy compared to euploidy at the same gestational age.

51 KEY WORDS: Placental-development; Chorionic villous; Trisomies; Metabolomics; Polyol 


\begin{abstract}
$54 \quad$ ABSTRACT
55

Objective: Placenta-related biological samples are used in biomedical research to investigate 57 placental development. Metabolomics represents a promising approach for studying placenta 58 placental metabolism in an effort to explain physiological and pathological mechanisms. The aAim 59 of the this study was to investigate metabolic changes in chorionic villous-villi during the first trimester of pregnancy in euploid and aneuploid cases.
\end{abstract}

Methods: Samples from 21 women (13 euploid, 8 aneuploid) were analyzed with ${ }^{1} \mathrm{H}-\mathrm{Nuclear}$ 62 Magnetic Resonance (NMR), Gas Chromatography-Mass Spectrometry (GC-MS) and High63 Performance Liquid chromatography (HPLC). Multivariate statistical analysis was performed and 64 differences in metabolites were used to identify the altered metabolic pathways. Results: A regression model to test the correlation between $\mathrm{CRL}$ fetal crown-rump length (CRL) 66 and metabolic profile of chorionic villous was performed in euploid pregnancies $\left(\mathrm{R}^{2}\right.$ was 0.69 for 67 the NMR analysis and 0.94 for the GC-MS analysis). Supervised Analysis was used to compare 68 chorionic villi of euploid and aneuploid fetuses (NMR: $R^{2} X=0.70, R^{2} Y=0.65, Q 2=0.30 R^{2} X=0.62$; 69 GC-MS: $\left.\mathrm{R}^{2} \mathrm{Y}=0.704, \mathrm{Q}^{2}=0.444\right)$. Polyol pathways, myo-inositol and oxidative stress seem to have a 70 fundamental role in euploid and aneuploid pregnancies.

71 Conclusion: Polyol pathways may have a crucial role in energy production in early pregnancy. 72 Excessive activation in aneuploid pregnancies may lead to increased oxidative stress. Metabolomics 73 represents a promising approach to investigate placental metabolic changes. 
80

81

82

83 84 85 86 87 88 89 90 91 92 93 94 95 96 97 98 99 100 53 54101 55 56102 57

\section{INTRODUCTION}

Placenta-related biological samples are used, in biomedical research, to investigate normal placental development, biology and pathophysiology ${ }^{1}$. In pregnancy, many changes in placenta development occur at the end of the first trimester ${ }^{2,3}$ : the oxygen tension within the intervillous space increases from $2.5 \%$ at 8 weeks to $8.5 \%$ at 12 weeks $^{4}$; the maternal metabolic profile changes from anabolic to catabolic metabolism to promote fetal growth, maturation and development ${ }^{5,6,7}$. During this gestational period, changes in maternal metabolic composition have, has been observed by the analysis of biofluids ${ }^{8}$ and similar changes could can also be observed also in placenta-placental tissue. This suggests suggesting physiologic mechanisms in both normal pregnancies and pathological changes as well, such as in cases of aneuploidy. Metabolomics represent a promising approach in the understanding of_the placental metabolism. Analytical techniques such as mass spectrometry $(\mathrm{MS})^{9}$ and nuclear magnetic resonance $(\mathrm{NMR})^{10}$ can provide information about tissue metabolites, such as lipids, amino acids and high-energy metabolites. This eould identifiedcan identify altered metabolic pathways ${ }^{11}$ providing a "snapshot" of the metabolic profile during different conditions $s^{12,13,14}$ and employing pattern recognition techniques ${ }^{15}$.

The metabolic profile of the placenta in early pregnancy is still poorly characterized ${ }^{16}$. Several studies identified differences in metabolomic profiles of serum markers in pregnant women with fetal chromosomal disorders ${ }^{17,18,19}$ but, to our knowledge, no study has been performed on chorionic villi collected after Transabdominal Chorionic Villus Sampling (TA-CVS).

The aim of our study was to evaluate differences in first trimester placenta metabolic pattern between euploid and aneuploid pregnancies through a metabolomic analysis of chorionic villi, obtained by TA-CVS.

\section{METHODS}


104 This prospective study was conducted in the Department of Prenatal Diagnosis in the 105 Microcitemico Pediatric Hospital in Cagliari, Sardinia. All patients underwent first-trimester 106 combined screening for aneuploidy between 11 weeks to 13 weeks and 6 days gestational age. First trimester combined test screening produces individualized risk estimates for trisomies 21,18 , and $13^{20}$ based on maternal age, ultrasound (used to document fetal nuchal translucency (NT) and fetal crown rump length $\left.\_,-\mathrm{CRL}\right)$ ) and biochemical parameters (maternal blood sampling determines biochemical markers including pregnancy-associated plasma protein A, PAPP-A, and free beta human chorionic gonadotropin, $\beta$-hCG). Combined screening is considered the most accurate method to produce an individual risk of aneuploidy and provides a detection rate of $95 \%$ and a false positive rate of $2.5 \%^{21}$. A cut-off risk of $1: 250$ for aneuploidies is high risk according to the Italian Society of Obstetric Gynecological Ultrasound (SIEOG) ${ }^{22}$. Such patients undergo genetic counseling and prenatal invasive diagnostic tests are offered.

All TA-CVS were performed between the $11^{\text {th }}$ and $14^{\text {th }}$ week of pregnaney gestation by free-hand transabdominal technique by a single operator $(\mathrm{GM})^{23}$. Written consent was obtained from all participating women, approved by Institutional Review Board of Microcitemico Hospital. After sampling, an adequate specimen of chorionic villi was used for cytogenetic examination and a remaining aliquot reserved for metabolomics analysis, was frozen immediately $(<2$ minutes $)$ in liquid nitrogen and kept at $-80^{\circ} \mathrm{C}$ until use.

Patient demographics (ethnic group, age), ultrasound data (e.g CRL, NT measurement), biochemical parameters (free $\beta-\mathrm{hCG}$ and PAPP-A) and fetal karyotype were collected.

Samples were divided inte two groups based on the outcome of the karyotype: euploid fetuses $(\mathrm{n}=13$, group EUPE) and aneuploid fetuses $(\mathrm{n}=8$, CHRgroup ANEUP). Aneuploidies-The aneuploid group included trisomy $21(\mathrm{n}=4)$, trisomy $18(\mathrm{n}=2)$ and trisomy $13(\mathrm{n}=2)$. Control patients were enrolled by choosing only those who underwent TA-CVS based only on their age (>35years). Moreover, some patients in the control group were offered an invasive procedure through genetic counselling because they had a history of a fetus with a chromosomopathy in a 
previous pregnancy. Patients showing abnormal combined screening test were not included in the

control group. The metabolomic profile of $C$-group EUP and CHR group ANEUP were then compared. The different aneuploidies were compared with each other and with the Control-control

\section{Groupgroup.}

\section{Sample preparation}

To standardize the protocol, we used the same amount of extraction solvent for all the samples. Briefly, CVS samples were mixed with $800 \mu \mathrm{L}$ of methanol and $200 \mu \mathrm{L}$ of Milli-Q water and then vortexed for 1 minutesminute. After 30 minutes of sonication in water with ice (Digital ultrasonic Cleaner, DU-32, Argo-Lab, Italy) samples were kept at $-20^{\circ} \mathrm{C}$ for 20 minutes and then centrifuged at 8,600 gfor $10 \mathrm{~min}$ at $4^{\circ} \mathrm{C}$. The supernatant containing low molecular weight compounds (e.g. sugars, fatty acids, amino acids) was collected for the metabolomics analysis. Concentrations of the metabolites in the samples were normalized after the analysis with NMR or GC-MS. Aliquots $(10 \mu \mathrm{l})$ from each sample were used to create a pool for quality control (QC) samples. A QC sample was injected at the beginning and at the end of the analysis. Subsequently, PCA (Principal Component Analysis) model was performed including the QC samples and based on their tight clustering, it showed a good quality of the analysis in our batch

\section{Nuclear Magnetic Resonance analysis and data processing}

For the NMR analysis, $600 \mu \mathrm{L}$ of the water-phase for each sample was dried overnight in a speedvacuum. The dried water-phase was re-suspended in $697 \mu$ of phosphate buffer $100 \mathrm{mM}$ in $\mathrm{D}_{2} \mathrm{O}$, pH 7.3 and $3 \mu \mathrm{l}$ of trimethylsilylpropanoic acid (TSP) $5.07 \mathrm{mM}$. TSP was added to provide an internal reference for the chemical shifts $(0 \mathrm{ppm})$, and $650 \mu \mathrm{l}$ of the solution were transferred to a 5 mm NMR tube.

The samples were analyzed with a Varian UNITY INOVA 500 spectrometer (Agilent Technologies, Inc., Santa Clara, CA, USA), which was operated at $499 \mathrm{MHz}$ equipped with a $5 \mathrm{~mm}$ triple resonance probe with $\mathrm{z}$-axis pulsed field gradients and an auto-sampler with 50 locations. 
155 One-dimensional ${ }^{1} \mathrm{H}-\mathrm{NMR}$ spectra were collected at $300 \mathrm{~K}$ with a pre-sat pulse sequence. The 156 spectra were recorded with a spectral width of $6000 \mathrm{~Hz}$; a frequency of $2 \mathrm{~Hz}$; an acquisition time of $1571.5 \mathrm{~s}$; a relaxation delay of $2 \mathrm{~ms}$; and a $90^{\circ}$ pulse of $9.5 \mu \mathrm{s}$. The number of scans was 512 . Each

10158 11 60

Free Induction Decay (FID) was zero-filled to $64 \mathrm{k}$ points and multiplied by a $0.5 \mathrm{~Hz}$ exponential line-broadening function. The spectra were manually phased and baseline corrected. By using MestReNova software (version 8.1, Mestrelab Research S.L.) each NMR spectrum was divided into consecutive "bins" of $0.04 \mathrm{ppm}$. The spectral area investigated was the region between 0.8 and 8.6 ppm. To minimize the effects of the different concentrations of chorionic villus samples, the integrated area within each bin was normalized to a constant sum of 100 . The final data set consisted of a $155 \times 21$ matrix.

\section{Gas-Chromatography Mass-Spectrometry analysis and data processing}

For GC-MS analysis, $300 \mu \mathrm{L}$ of each extract were dried with a vacuum concentrator overnight (Eppendorf concentrator plus, Eppendorf AG, Hamburg, Germany)and were derivatized with $25 \mu \mathrm{L}$ of methoxyamine dissolved in pyridine $(10 \mathrm{mg} / \mathrm{mL})$ (Sigma-Aldrich, St. Louis, MO, USA) at $70^{\circ} \mathrm{C}$. After $1 \mathrm{~h}, 50 \mu \mathrm{L}$ of N-Methyl-N-(trimethylsilyl)-trifluoroacetamide, (MSTFA, Sigma-Aldrich, St. Louis, MO, USA) was added and samples were left at room temperature for one hour. Samples were diluted in $50 \mu \mathrm{L}$ of hexane (Sigma-Aldrich, St. Louis, MO, USA) and one microliter of derivatized sample was injected splitless into a 7890A gas chromatograph coupled with a 5975C Network mass spectrometer (Agilent Technologies, Santa Clara, CA, USA) equipped with a $30 \mathrm{~m}$ $\times 0.25 \mathrm{~mm}$ ID, fused silica capillary column, with a $0.25 \mu \mathrm{M}$ TG-5MS stationary phase (Thermo Fisher Scientific, Waltham, MA, USA). The injector and transfer line temperatures were at $250^{\circ} \mathrm{C}$ and $280^{\circ} \mathrm{C}$, respectively. The gas flow rate through the column was $1 \mathrm{ml} / \mathrm{min}$. The column initial temperature was kept at $60{ }^{\circ} \mathrm{C}$ for $3 \mathrm{~min}$, then increased to $140^{\circ} \mathrm{C}$ at $7^{\circ} \mathrm{C} / \mathrm{min}$, held at $140^{\circ} \mathrm{C}$ for 4 min, increased to $300^{\circ} \mathrm{C}$ at $5^{\circ} \mathrm{C} / \mathrm{min}$ and kept for $1 \mathrm{~min}$. For the analyzed samples we extracted masses from a range 50-600 m/z. Identification of metabolites was performed using the standard 
181 Library-2007) and GMD (http://gmd.mpimp-golm.mpg.de) mass spectra libraries (match $\geq 40 \%$ ) 182 and, when available, by comparison with authentic standards. Data processing was performed by 183 using a pipeline in Knime ${ }^{24} \underline{\mathrm{KNIME}^{24}}$. In brief, peak detection and deconvolution were performed in a R-XCMS package, filtering was performed using blank samples and keeping features present in $\geq 50 \%$ of the samples. Missing value imputation was conducted by using random forest algorithm. Relative concentrations of the discriminant metabolites were obtained by the chromatogram area and then normalized by median fold change. All the parameters were reported in supplementary materials.

\section{Determination of intracellular aminothyol levels}

Glutathione reduced and oxidized (GSH, GSSG) and ascorbic acid levels were determined in chorionic villi, using a modified method described by Khan et al $^{25}$. Samples were dissolved in 150 $\mu \mathrm{l}$ of $10 \%$ meta-phosphoric acid solution. After vortexing for 2 minutes, $150 \mu \mathrm{l}$ of $0.05 \%$ TFA (trifluoroacetic acid) solution was added and centrifuged for $10 \mathrm{~min}$ at $10000 \mathrm{rpm}$ at $4{ }^{\circ} \mathrm{C}$. An aliquot was transferred in-to an Eppendorf tube for the determination of the proteins through Bradford Assay. The supernatant was injected into the HPLC system (Agilent 1260 infinity, Agilent Technologies, Palo Alto, USA). GSH, GSSG and ascorbic acid levels were measured by electrochemical detection ${ }^{26}$, using an HPLC coupled with an electrochemical detector (DECADE II Antec, Leyden, The Netherlands) and an Agilent interface 35900E. A C-18 Phenomenex Luna column, $5 \mu \mathrm{m}$ particle size, $150 \times 4.5 \mathrm{~mm}$, was used with a mobile phase of $99 \%$ water with $0.05 \%$ TFA (v/v) and $1 \% \mathrm{MeOH}$ at a flow rate of $1 \mathrm{ml} / \mathrm{min}$. An eElectrochemical detector was set at an oxidizing potential of $0.74 \mathrm{~V}$. Data were collected and analysed using the Agilent Chemstation A.10.02 Software, and expressed as area of GSH, GSSG and ascorbic acid peak to $\mu$ g of proteins.

\section{Statistical analysis}


204 Multivariate statistical analysis was performed on NMR and GC-MS data by using SIMCA-P 205 software (ver. 14.0, Umetrics, Sweden) ${ }^{27}$. The variables were Pareto scaled for the ${ }^{1} \mathrm{H}-\mathrm{NMR}$ 206 analysis and UV scaled for the GC-MS analysis.

The initial data analyses were conducted using the Principal Component Analysis (PCA) for the exploration of the sample distributions without classification. To identify potential outliers, the DmodX and Hotelling's T2 tests were applied.

A supervised analysis was subsequently used. Orthogonal Partial Least Square (OPLS-DA) analysis and Partial Least Square (PLS-DA) maximize the discrimination between samples assigned to different classes. The variance and the predictive ability $\left(\mathrm{R}^{2} \mathrm{X}, \mathrm{R}^{2} \mathrm{Y}, \mathrm{Q}^{2}\right)$ were established to evaluate the suitability of the models. Since VIP (Variable Influence on Projection) $>1$ are the most relevant for explaining Y (assignment of two classes) ${ }^{27}$, our OPLS-DA model for the NMR matrix and PLS-DA models for the GC-MS matrix were performed by using only variables corresponding to VIP value $>1$. In addition, a permutation test $(n=400)$ was performed to validate the models. The scores from each PLS-DA model were subjected to a CV-ANOVA to test for significance $(p<0.05)$. To study a possible linear relationship between a matrix Y (dependent variables, e.g. clinical parameters such as length of the fetus) and a matrix X (predictor variables, e.g. metabolites) Partial Least Squares projection to latent structures regression (PLS) model was performed ${ }^{28}$.

The most significant variables were extracted by the loading plot from the PLS-DA model and from the S-plot from the OPLS-DA model and for the ${ }^{1} \mathrm{H}-\mathrm{NMR}$ data were identified using the Chenomx NMR Suite 7.1 (Chenomx Inc., Canada) ${ }^{29}$ and on literature data. GraphPad Prism software (version 7.01, GraphPad Software, Inc., CA, USA) was used to perform the univariate statistical analysis of the data resulting from the multivariate analysis and from the HPLC analysis. To verify the

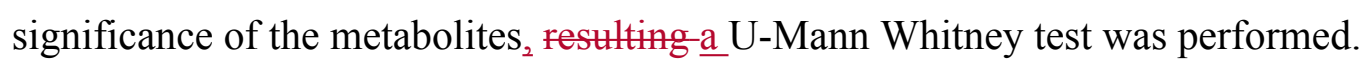

\section{Pathways analysis}


229 Metabolic pathways were generated by using MetaboAnalyst 3.0 $0^{30}$, a web server designed to obtain 230 a comprehensive metabolomic data analysis, visualization and interpretation ${ }^{31}$. This approach 231 permits correlation of metabolite changes with metabolic networks. The pathway analysis module of Metaboanalyst $3.0^{30,31}$ uses the high-quality KEGG metabolic pathways as the backend knowledgebase. Literature was also used to identify the most important pathways involved.

\section{RESULTS}

\section{Metabolic changes in association with CRL}

In this study, 21 samples of chorionic villi (13 controls euploid and 8 aneuploid) were analyzed with ${ }^{1} \mathrm{H}-\mathrm{NMR}$ and HPLC while 17 (9 controls, 4 controls samples were not analyzed for lack of biological sample, and 8 chromosomal disorders) were analyzed by GC-MS. The number of metabolites identified were: 47 with ${ }^{1} \mathrm{H}-\mathrm{NMR}$ and 28 with GC-MS, including organic acids, amino acids, fatty acids and sugars (Fig. 1 A-B).

To investigate a possible correlation between the metabolic profile of chorionic villous in euploid pregnancies and the CRL (a specific marker of gestational age), PLS regression analysis was performed (Fig. 2 A-B) using the matrices resulting from both NMR and GC-MS analysis.

The correlation analysis showed a $\mathrm{R}^{2}=0.69$ for the NMR analysis and $\mathrm{R}^{2}=0.94$ for the GC-MS analysis. The correlation between the NMR-metabolic profile and the CRL parameter indicated a linear positive correlation with increasing concentrations of myo-inositol, glutamine and citrate. Looking at the distribution of metabolites in the analysis of the GC-MS matrix, there was a positive linear correlation with the concentration of myo-inositol, inositol, glycerol, dehydroascorbic acid and ribitol, while an inverse correlation with the concentration of xylitol, 1,5-anydro-D-Sorbitol, Dfructose and D-mannose was observed.

\section{Metabolic fingerprinting of chromosomal disorders}

Subsequently, to investigate any differences between euploid and aneuploid samples of the same gestational age, a PLS-DA analysis was performed (Fig. 2 C-D) and the obtained model showed the following statistical parameters: $\mathrm{R}^{2} \mathrm{X}=0.70, \mathrm{R}^{2} \mathrm{Y}=0.65, \mathrm{Q}^{2}=0.30$, for the NMR analysis (Fig. 2C) 
and $\mathrm{R}^{2} \mathrm{X}=0.60, \mathrm{R}^{2} \mathrm{Y}=0.75, \mathrm{Q}^{2}=0.47$ for the GC-MS analysis (Fig. 2D). The models were then validated with the permutation test, (for the NMR, $\mathrm{R}^{2}$ intercept $=0.428$; $\mathrm{Q}^{2}$ intercept $=-0.12$, for the GC-MS, $\mathrm{R}^{2}$ intercept $=0.47 ; \mathrm{Q}^{2}$ intercept $=-0.6$ ).

The multivariate analysis identified a unique pattern of metabolites in the aneuploid chorionic villi. In particular, NMR analysis displayed an increase of lactate, asparagine, branched-chainaminoacids (valine, leucine and isoleucine) and a decrease of myo-inositol, glycerol, fumarate, betaine and acetate in CHR group ANEUP compared to group EUPC. At the same time, GC-MS analysis showed that eleven metabolites were responsible for the separation between the groups. In particular, D-sorbitol, 1,5-anydro-D-sorbitol, D-fructose, dehydroascorbic acid and glucose were increased in CHR Groupgroup ANEUP, while cholesterol, pyruvic acid, palmitic acid, inositol, homoserine and stearic acid were decreased. The bar graphs representing the mean concentration of the metabolites and the relative standard deviations are shown in Fig. 3.

The metabolites having-with VIP value $>1$ resulting from the multivariate analysis, were used to identify the most important metabolic pathways involved in chromosomal diseases(Fig. 4A).

The most perturbed pathways were involved in energetic processes, such as glycolysis and gluconeogenesis, pentose phosphate shunt, pyruvate metabolism and TCA cycle. Statistical parameters of the pathways analysis (p-value, $\mathrm{n}^{\circ}$ metabolites involved for each pathway) are reported in Table 1S. Several of the metabolites which differed in the multivariate analysis were involved in the polyol pathway (glucose, fructose, sorbitol), suggesting a hypothetic role of this process in chromosomal disorders (Fig. 4B).

The excessive activation of the polyol pathway proposed in CHRgroup ANEUP, suggests the presence of an oxidative stress environment. This data was confirmed with the analysis of the amino thiols levels: GSH and GSSG- appear to decrease in CHRgroup ANEUP. A similar observation was found for ascorbic acid level, which was significantly lower in CHR group ANEUP(Fig. 3B). 
Subsequently, to understand the metabolic differences between controls and each individual aneuploidy, supervised PLS-DA models were performed both with the data of NMR (data not shown) and GC-MS (Fig. 5A). Statistical parameters were: C group EUP vs Trisomy 21_(group $\underline{E U P=7,}$ Trisomy $21=4$ ), $\mathrm{R}^{2} \mathrm{X}=0.62, \mathrm{R}^{2} \mathrm{Y}=0.887, \mathrm{Q}^{2}=0.441$, (permutation test-: $\mathrm{R}^{2}$ intercept $=0.79$; $\mathrm{Q}^{2}$ intercept $=0.06$ ) $;$, C group EUP $v s$ Trisomy 18 (group EUP=7, Trisomy 18=2): $\mathrm{R}^{2} \mathrm{X}=0.509$, $\mathrm{R}^{2} \mathrm{Y}=0.940, \mathrm{Q}^{2}=0.431$ (permutation test-: $\mathrm{R}^{2}$ intercept $=0.78 ; \mathrm{Q}^{2}$ intercept $=0.05$ ), -group EUP $v s$ Trisomy 13 (group EUP=7, Trisomy $13=2$ ): $\mathrm{R}^{2} \mathrm{X}=0.557, \mathrm{R}^{2} \mathrm{Y}=0.868, \mathrm{Q}^{2}=0.399$ (permutation test-: $\mathrm{R}^{2}$ intercept $=0.77 ; \mathrm{Q}^{2}$ intercept $\left.=0.01\right)$. Based on the PLS-DA models, aA global overview of the different metabolic profiles of the three chromosomal diseases is summarized in Fig. 5B. The results show the overall potential of the metabolomics approach; however, due to the small number of samples for each individual aneuploidy, additional study is necessary (as evidenced by the permutation tests).

\section{DISCUSSION}

In this study, a metabolomics approach was applied to describe the normal metabolic status of placenta in the first trimester of pregnancy and to compare euploid and aneuploid chorionic villi samples. Despite the low number of the-samples (due to the fact that it is not easy to enroll a large number of patients with aneuploid fetuses-affected by trisemies is not easy), chorionic villous samples, collected-for-the karyotype analysis, were analyzed through three different analytical techniques (NMR, GC-MS and HPLC) in order to have an overview of the placenta metabolome as complete as possible. Pregnancy is a period characterized by several physiological changes in metabolic, biochemical, hormonal and immunological status ${ }^{32}$. For this reason, metabolic alterations of chorionic villus in the crucial period between the $11^{\text {th }}$ to $14^{\text {th }}$ week of pregnancy, were investigated.

\section{Metabolic changes in association with CRL}


304 Normal pregnancies are characterized by changes in the levels of haematological circulating free 305 fatty acids, triglycerides, cholesterol and phospholipids and a-insulin resistance ${ }^{33}$. These metabolic 306 changes represent a necessary and indispensable adaptation to satisfy the fetal energy demands

necessary for rapid growth and to prepare the maternal body system-for delivery and lactation ${ }^{34}$. Insulin resistance, a physiologic change during pregnancy, is the basis of this metabolic maternal adaptation to ensure adequate fetal carbohydrate supply.

As pregnancy advances, insulin sensitivity gradually declines to $50 \%{ }^{35}$ due to hormones (estrogen, progesterone) and the activity of other factors, such as inositol and myo-inositol ${ }^{36}$. Inositol and myoinositol are intracellular mediators of the insulin signal; theyand are correlated with insulin sensitivity and, indeed they could be implicated in glucose homeostasis $37,38,39$.

Our results demonstrated changes in the concentrations of some metabolites in chorionic villi correlating with the fetus length. Particularly, myo-inositol and inositol levels linearly correlate with the CRL, both in the NMR and GC-MS analysis, suggesting a biological role during pregnancy. The correlation of these metabolites with CRL supports involvement in glucose homeostasis as several studies have previously reported ${ }^{33}$.

Cholesterol levels in chorionic villi, as well as myo-inositol and inositol, were increased in correlation with the CRL. The higher concentration of cholesterol may the result of increased levels of pro-gestational hormones. In_fact ${ }_{2}$ in -maternal blood cholesterol represent represents the precursor of both progesterone and estrogen ${ }^{40}$.

Aminoacids are essential factors for fetal development and growth. T, they arerepresent the precursors for the biosynthesis of macromolecules (proteins and nucleotides), signalling functions and Adenosine triphosphate (ATP) production $^{41}$. In our data, glutamine showed a linear correlation with the CRL and gestational age. Glutamine is involved in numerous physiological metabolic pathway of the fetus ${ }^{42,43}$. It is a substrate for protein synthesis and an anabolic precursor for muscle growth. Glutamine is a precursor for neurotransmitters, for nucleotide and nucleic acid synthesis and for glutathione production ${ }^{41,43}$. 
330 Finally, our data revealed that there is an increased concentration of xylitol, 1,5-anydro-D-sorbitol, 331 D-fructose and D-mannose in the early stages of the first trimester of pregnancy. These metabolites 332 are known to be involved in the polyol pathway, which is susceptible to glucose concentration.

Polyols are polyhydric alcohols formed by the reduction of aldoses and ketoses, and their precursors are essential substrates for the glycolytic and pentose phosphate pathways. It has been hypothesized that polyols are the first source of carbohydrates for early life ${ }^{44}$ and this could explain the high level of these metabolites seen in our first trimester placenta samples.

The polyol pathway starts with glucose, which normally enters into glycolysis to produce pyruvic acid and acetyl-CoA. Insulin is one of the key regulators of metabolism ${ }^{45}$. Indeed, maternal insulin resistance plays an important role in the regulation of maternal energy metabolism, fat deposit and fetal growth ${ }^{46,47}$. In a hyperglycemic environment, increased intracellular glucose results in its increased enzymatic conversion to the polyalcohol sorbitol, with a concomitant decrease in NADPH. NADPH is required for regenerating reduced glutathione (GSH) and this might induce or exacerbate intracellular oxidative stress $^{48}$.

Furthermore, in the first trimester of pregnancy, the oxygen tension within the intervillous space increases from $2.5 \%$ at 8 weeks to $8.5 \%$ at 12 weeks ${ }^{2}$. Metabolism of the placenta tissue appears to adapt to these conditions through the activation of the polyol pathway. This pathway could provide an important mechanism for the re-oxidation of pyridine nucleotides under conditions of low oxygenation, enabling glycolysis to continue without an excessive rise in acidity ${ }^{2}$.

\section{Metabolic fingerprinting of chromosomal disorders}

The polyol pathway appeared to be modified when comparing samples fromof -euploid and aneuploidy aneuploid chorionic villi with abnormal and normal chromosomes at the same gestational age. Metabolites such as D-sorbitol (vip scores $>1$ ), D-fructose (vip scores $>1$ ) , Dglucose $(p<0.05)$ and pyruvic acid (vip scores $>1)$ were found to be increased in the group ANEUPCHR group suggesting an over activation of the polyol pathway accompanied by a significant decrease of myo-inositol $(\mathrm{p}<0.05)$. 
The increase of intracellular oxidative stress in CHR the group ANEUP, resulting fromby the activation of this pathway, was confirmed by HPLC analysis. A decrease of GSH (although not statistically significant), ascorbic acid $(\mathrm{p}<0.05)$ and an increase of dehydroascorbic acid $(\mathrm{p}<0.05)$ were also found. Despite the possible presence of oxidative stress discussed above, we didn't ebserveddid not observe an increase of GSSG as we expected. The concentration of these metabolites confirms the increase of the intracellular oxidative stress and ${ }_{2}$ consequently, the depletion of antioxidant defenses. Alterations in these pathways and the resultant excess oxidative stress, might represent be a primary pathological features-feature in trisomies.

These-Our results showed a decrease of stearic and palmitic acid $(\mathrm{p}<0.05)$, in CHR Groupthe group

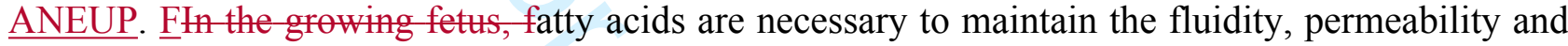
conformation of the membranes of the growing fetus and are essential as a source of energy and as precursors of important bioactive compounds such as the prostacyclins, prostaglandins, thromboxanes and leukotrienes. Apart from the overall placental supply of fatty acids, there is some evidence that the composition of the fatty acids may affect fetus-fetal growth ${ }^{49}$.

This analysis showed decreased levels of cholesterol_levels $(\mathrm{p}<0.05)$ in villi samples from the three chromosomal disorders studied here. Cholesterol is a major structural lipid of cell membranes, as well as the precursor of steroid hormones and bile acids. It was previously reported that cholesterol levels are significantly decreased in trisomies 18 and 21 compared to controls in plasma ${ }^{50}$ and amniocytes $^{51}$. Our findings extend these results also to chorionic villi and likely reflect a disruption of the cholesterol pathway.

In conclusion, this study highlights a novel tool for studying metabolic alterations in placental tissue obtained during TA-CVS. Differences in metabolic concentrations were observed by gestational age and in the presence of aneuploidy. Finally, the metabolomics approach ean-may be able to discriminate the three common trisomies based on the metabolic profile, but further analysis is necessary because the number of samples of all three trisomies was too low. This is Despite the new topic of this scientific work, this represents-a preliminary study of metabolomics analysis in first 
382 trimester TA-CVS samples-due to the fact that the aneuploidies have a low incidence and further 383 investigations are required. . Considering the low incidence of aneuploidies, further investigations are required before drawing conclusive comparisons between them.

\section{Data availability statement}

The data that support the findings of this study are available from the corresponding author upon reasonable request.

Tables

Table 1. Demographic and-clinical features of patients - with feti-affected by chromosomat disorders and controls

\begin{tabular}{|c|c|c|c|c|c|}
\hline \multirow{6}{*}{} & \multicolumn{5}{|c|}{ Patients information } \\
\cline { 2 - 6 } & $\mathrm{N}$ & $\begin{array}{c}\text { Age } \\
\text { (Average } \pm\end{array}$ & $\begin{array}{c}\text { NT } \\
\text { (Average } \pm\end{array}$ & CRI & BMH \\
\hline
\end{tabular}


3

\begin{tabular}{|c|c|c|c|c|c|}
\hline & & SD) & SD) & (Average \pm SD) & \\
\hline Controls & 13 & $36.07 \pm 4.9$ & $2.12 \pm 1.67$ & $59.10 \pm 10.6$ & 19.6 \\
\hline Aneuploids & 8 & $35.75 \pm 6.2$ & $3.98 \pm 0.5$ & $60.31 \pm 9.7$ & 20.8 \\
\hline
\end{tabular}

Abbreviations. NT= Nuchal Translucency; CRL=Crown Rump Length, BMI= Body Mass Index.

Table 2. Panel of the most discriminant metabolites resulting from the multivariate analysis. In the table were included the VIP value (for the metabolites assayed with ${ }^{-}{ }^{1}$ HNMR and GC MS), the pvalue (U-Mann Whitney test) with the respective correction for multiple comparisons (BenjaminiHochberg) for each metabolite.

\begin{tabular}{|c|c|c|c|c|}
\hline & \\
\hline & Variables & VIP value & P-value & $\begin{array}{l}\text { Benjamini- } \\
\text { Hochberg }\end{array}$ \\
\hline \multirow{10}{*}{$\frac{\alpha}{2}$} & Myo-Inositol & 1.44293 & 0.01 & 0.036 \\
\hline & tactate & 1.18766 & 0.1 & - \\
\hline & Asparagine & 1.87244 & 0.3 & - \\
\hline & Glycerol & 1,00025 & 0.4 & - \\
\hline & Valine & 1,52049 & 0.8 & $2-$ \\
\hline & Leucine & 1,11058 & 0.02 & 0.036 \\
\hline & Isoleucine & 1,49307 & $0.0 z$ & 0.036 \\
\hline & Fumarate & 1.05523 & 0.32 & - \\
\hline & Betaine & 1,09841 & 0.16 & - \\
\hline & Acetate & 1.02471 & 0.07 & - \\
\hline \multirow{8}{*}{$\underset{4}{4}$} & Pyruvate & 1,96127 & 0.2 & - \\
\hline & Homoserine & 1,02222 & 0.5 & - \\
\hline & Dehydroascorbic acid & 1,19049 & 0.03 & 0.038 \\
\hline & D-fructose & 1,04314 & 0.07 & - \\
\hline & 1,5-anydro-D-sorbitol & 1,08823 & 0.1 & - \\
\hline & Glucose & 1,22809 & 0.04 & 0.045 \\
\hline & d-Sorbitol & 1,18827 & 0.09 & - \\
\hline & Inositol & 1,22475 & 0.08 & - \\
\hline
\end{tabular}




\begin{tabular}{|c|c|c|c|c|}
\hline & Palmitic acid & 1,31942 & 0.02 & 0.036 \\
\hline & Stearic acid & 1,24499 & 0.05 & NS \\
\hline & Cholesterol & 1,57857 & 0.004 & 0.036 \\
\hline \multirow{3}{*}{$\frac{4}{\underline{a}}$} & GSSH & - & 0.11 & - \\
\hline & GSH & - & 0.23 & - \\
\hline & Vit. C & - & 0.03 & 0.038 \\
\hline
\end{tabular}

\section{Figures}

Figure 1. Identified compounds in chorionic villous samples analyzed with NMR and GC-MS.

Figure 1A. Identified compounds in NMR representative spectrum:1. Lipid1; 2. Isoleucine; 3. Leucine; 4. Valine; 5. Lipid 2; 6. Lactate; 7. Threenine; 8. Alanine; 9. Lysine; 10. Thymine; 11. Acetate; 12. Proline; 13. Glutamate; 14. Methionine; 15. Glutamate; 16. Pyroglutamate; 17. Glutamine; 18. Citrate; 19. aspartate; 20. Sarcosine; 21. Asparagine; 22. Creatine; 23; Ornithine; 24. Ethanolamine; 25. Choline; $26-O$ phosphocholine; 27. Glycero phosphocholine; 28. Glucose; 29. Betaine; 30. Myo-inositol; 31. Glycine; 32. Glycerol; 33. Glucitol; 34. Serine; 35. Fructose; 36. Nucleotides; 37. Mannose; 38. Uracil; 39. Fumarate; 40. Tyrosine; 41. Histidine; 42. n-Methylhistidine; 43. Phenylalanine; 44. Tryptophan; 45. Xanthine; 46. Hypoxanthine; 47. Formate.

B. Identified compounds in GC-MS representative chromatogram: 1. Pyruvic acid; 2. Lactic acid; 3. L valine; 4. L-alanine; 5. Leucine; 6. L-isoleucine; 7. Urea; 8. Ethanolamine; 9. L-serine; 10. Glycerol; 11. Glycine; 12. Succinic acid; 13. Fumaric acid; 14. Malic acid; 15. Homoserine; 16. Xylitol; 17. Ribitol; 18. Dehydreacorbic acid; 19. 1,5-anydro-D-sorbitol; 20. D-fructose; 21. D-glucose; 22. D-sorbitel; 23. DMannitol; 24. Palmitic acid; 25. Inositel;, 26. Myo-inositol; 27. Stearic acid; 28. Cholesterol

Figure 2. Partial least square regression analysis and partial least square discriminant analysis.

Figure 2. A-B PLS regression analysis. Correlation between the metabolic profile of chorionic villous and CRL parameter of fetuses (points represent control samples (Group C) and the color indicated the respective (RL). The correlation analysis showed a $R^{2}=0.69$ for the NMR analysis and $R^{2}=0.94$ for the GCMS 
analysis. C-D. PLS-DA models of samples with controls (Group C) and chromosomal disease (Group CHR). For the NMR analysis (C) statistical parameters were $R^{2} X=0.70, R^{2} Y=0.65, Q^{2}=0.30$, while for the GC-MS analysis (D) were $R^{2} X=0.60, R^{2} Y=0.75, Q^{2}=0.47$. The models were then validated with the

Figure 3. Comparison of select metabolites assayed in chorionic villous of fetuses with normal and abnormal karyotype.

Figure 3. The most important metabolites espressed by ranks with standard deviations as determined by different techniques. Group $C$ were cases with normal chromosomes and Group CHR were cases with abnormal chromosomes. Statistical analysis were performed by Mann-Whitney Utest. Metabolites differences between groups with $p$-value $<0.05$ are indicated with the star. A)Analysis by NMR. B) Analysis by GC-MS C) Analysis by HPLC: box plots of the intracellular aminothiols such as GSH, GSSG and ascorbic acid. Data are expressed as area of peak to $\mu \mathrm{g}$ of proteins

\section{Figure 4. Pathways analysis.}

Figure-4. Most important pathways involved in placentas in cases with abnormal chromosomes. A) Summary of the most important pathways resulting from the analysis with Metaboanalyst: TCA cycle, pyruvate metabolism, glycolysis and gluconeogenesis, pentose phosphate pathway, glycine, serine and threonine metabolism were the most involved nets. B) Roles of the identified metabolites (green arrows means increase while blue arrows decrease) involved in the polyols pathway.

Figure 5. Partial least square diseriminant analysis of trisomy 21, trisomy 18, trisomy 13 and control Group. 
454 Figure 5A. PLS-DA models of the three different chromosomal diseases (21, 18 and 13) and control group 455 built with the GCMS data (C). Statistical parameters were $C$ vs Trisomy $21, R^{2} X=0.62, R^{2} Y=0.887$, $456 Q^{2}=0.441, C$ vs Trisomy 18: $R^{2} X=0.509, R^{2} Y=0.940, Q^{2}=0.431, C$ vs Trisomy $13: R^{2} X=0.557, R^{2} Y=0.868$, $457 \quad Q^{2}=0.399$ B. Venn diagram showing metabolite relationships between trisomy 21, trisomy 18 and trisomy 13 458 is shown. 


\section{REFERENCES}

[1] G.J. Burton, N.J. Sebire, L. Myatt, et al. Optimising sample collection for placental research. Placenta. 2014; 35:9-22.

[2] T. Cindrova-Davies, M. Tissot van Patot, L. Gardner, et al. Energy status and HIF signalling in chorionic villi show no evidence of hypoxic stress during human early placental development. Mol Hum Reprod. 2015; 21: 296-308.

[3] G.J. Burton, E. Jauniaux, A.L. Watson. Maternal arterial connections to the placental intervillous space during the first trimester of human pregnancy: the Boyd collection revisited. Am J ObstetGynecol. 1999; 181:718-724.

[4] E. Jauniaux, J. Hempstock, N. Greenwold, G.J. Burton. Trophoblastic oxidative stress in relation to temporal and regional differences in maternal placental blood flow in normal and abnormal early pregnancies. Am J Pathol. 2003; 162:115-125.

[5] K.Y. Lain et al. Metabolic Changes in Pregnancy. Clinical Obstetrics and Gynecology. 2007; 50:938-948.

[6] E. Herrera, H. Ortega-Senovilla. Lipid Metabolism During Pregnancy and its Implications for Fetal Growth. Current pharmaceutical biotechnology. 2014;15(1).

[7] Z. Zeng, F. Liu, S. Li. Metabolic Adaptations in Pregnancy: A Review. Ann Nutr Metab. 2017;70:59-65.

[8] M. Orczyk-Pawilowicz, E. Jawien, S. Deja, et al. Metabolomics of Human Amniotic Fluid and Maternal Plasma during Normal Pregnancy. PLOS ONE. 2016; 0152740.

[9] K. Dettmer, P.A. Aronov, B.D. Hammock, Mass spectrometry-based metabolomics. Mass Spectrom Rev. 2007; 26:51-78.

[10] J.K. Nicholson, J.C. Lindon, E. Holmes. 'Metabonomics': understanding the metabolic responses of living systems to pathophysiological stimuli via multivariate statistical analysis of biological NMR spectroscopic data. Xenobiotica. 1999; 29:1181-1189. 
[11] D.S. Wishart, D. Tzur, C. Knox. HMDB: the Human Metabolome Database. Nucleic Acids Res. 2007; 35:D521-D52.

[12] D.I. Ellis, W.B. Dunn, J.L. Griffin, J.W. Allwood, R. Goodacre. Metabolic fingerprinting as a diagnostic tool. Pharmacogenomics. 2007; 8:1243-1266.

[13] S. Poddighe, F. Murgia, L. Lorefice, et al. Metabolomic analysis identifies altered metabolic pathways in Multiple Sclerosis. Int J of Bioch and Cell Biology. 2017; 93: 148-155.

[14] Iuculano, A., Murgia, F., Peddes, C., et al.. Metabolic characterization of amniotic fluids of fetuses with enlarged nuchal translucency. Journal of Perinatal Medicine.2019, doi:10.1515/jpm-2018-0314.

[15] N.J. Serkova, C.U. Niemann. Pattern recognition and biomarker validation using quantitative 1H-NMR-based metabolomics. Expert. Rev. Mol. Diagn. 2006; 6:717-731.

[16] W.B. Dunn, M. Brown, S.A. Worton, et al. The metabolome of human placental tissue: investigation of first trimester tissue and changes related to preeclampsia in late pregnancy. Metabolomics. 2012;8:579-597.

[17] R.O. Bahado-Singh, R. Akolekar, R. Mandal et al. Metabolomic analysis for first-trimester Down syndrome prediction. Am J Obstet Gynecol. 2013;208:371.

[18] R.O. Bahado-Singh, R. Akolekar, R. Mandal et al. Metabolomic prediction of trisomy 18 first trimester pregnancies. Am J ObstetGynecol, 2013; 208(1).

[19] J. Pinto, L.M. Almeida, A.S. Martins et al. Impact of fetal chromosomal disorders on maternal blood metabolome: toward new biomarkers? Am J Obstet Gynecol. 2015;213:841

[20] K.H. Nicolaides, D. Wright, C. Poon, A. Syngelaki. M.M. Gil. First-trimester contingent screening for trisomy 21 by biomarkers and maternal blood cell-free DNA testing. Ultrasound Obstet Gynecol. 2013; 42: 41-50.

[21] K.H. Nicolaides. Screening for fetal aneuploidies at 11 to 13 weeks. PrenatDiagn. 2011; 31: $7-15$. 
[22] Società italiana di Ecografia Ostetrico Ginecologica (SIEOG). Linee Guida SIEOG EDITEAM; 2015: 15-19.

[23] G. Monni, R. Ibba. R. Lai, et al. Early transabdominal chorionic villus sampling in couples at high genetic risk, American Journal of Obstetrics and Gynecology. 1993; 168:170-173.

[24] S. Liggi, C. Hinz, Z. Hall, Et al. KniMet: A pipeline for the processing of chromatographymass spectrometry metabolomics data. Metabolomics. 2018; 14, 52.

[25] A. Khan, M. Khan, Z. Iqbal, et al. A new HPLC method for the simultaneous determination of ascorbic acid and aminothiols in human plasma and erythrocytes using electrochemical detection.Talanta. 2011; 84:789-801.

[26] K.A. Veselkov, L.K. Vingara, P. Masson, et al. Optimized preprocessing of ultraperformance liquid chromatography/mass spectrometry urinary metabolic profiles for improved information recovery. Anal Chem. 2011;83:5864-72.

[27] L. Eriksson, T. Byrne, E. Johansson, et al. Multi-and Megavariate Data Analysis, Basic principles and applications. Umetrics Academy, Malmo, Sweden. 2013; 1-501.

[28] S. Wold, L. Eriksson, PLS_regression: a basic tool oh chemometrics, journal of chemometrics. 2001b; 58:109-30.

[29] A.M. Weljie, J. Newton, P. Mercier, et al. Targeted profiling: quantitative analysis of ${ }^{1} \mathrm{H}$ NMR metabolomics data. Anal Chem. 2006; 78:4430-4442.

[30] www.metaboanalyst.ca

[31] J. Xia, I. Sinelnikov, H. Beomsoo, D. Wishart. MetaboAnalyst 3.0-making metabolomics more meaningful. Nucleic Acids Res. 2015; 43: W251-W257.

[32] F. Cunningham, K. Lenevo, S. Bloom, et al. Maternal physiology. Williams Obstetrics, 22nd ed. USA: McGraw Hill; 2005. pp107-31.

[33] P. Catalano, N. Roman-Drago, S. Amini, E. Sims. Longitudinal changes in body composition and energy balance in lean women with normal and abnormal glucose tolerance during pregnancy. Am J Obstet Gynecol. 1998; 179:156-65. 
[34] G. Di Cianni, A. Ghio, V. Resi, L. Volpe. Gestational Diabetes Mellitus: an Opportunity to Prevent Type 2 Diabetes and Cardiovascular Disease in Young Women. Women's Health. 2010; 6:97-105.

[35] K. McLachlan, D. O’Neal, A. Jenkins, F. Alford. Do adiponectin, TNF $\alpha$, leptin and CRP relate to IR in pregnancy? Studies in women with and without gestational diabetes, during and after pregnancy. Diabetes Metab Res Rev. 2006; 22:131-38.

[36] E.A. Ryan, L. Enns. Role of gestational hormones in the induction of IR. J ClinEndocrinolMetab. 1988; 67:341-47.

[37] S. Suzuki, H. Kawasaki, Y. Satoh. Urinary chiro-inositol excretion is an index marker of insulin sensitivity in Japanese type II diabetes. Diabetes Care. 1994; 17:1465-1468.

[38] J. Larner, D.L. Brautigan, M.O. Thorner. d-Chiro-inositol glycans in insulin signaling and insulin resistance. Mol Med. 2010;16:543-51.

[39] F. Corrado, R. D'Anna, G. Di Vieste, et al. The effect of myoinositol supplementation on insulin resistance in patients with gestational diabetes. Diabet Med. 2011;28:972-5.

[40] Human Physiology: From Cells to Systems, 2013, 2010, Books/Cole, Cengage learning.

[41] G. Wu, F.W. Bazer, G.A. Johnson, et al. Maternal and fetal amino acid metabolism in gestating sows. SocReprodFertil. 2013; 68:185-198.

[42] X. Wu, C. Xie, Y. Zhang, et al. Glutamate-glutamine cycle and exchange in the placentafetus unit during late pregnancy. Amino Acids. 2015; 47:45-53.

[43] P. Newsholme, M.M. Lima, J. Procopio,et al. Glutamine and glutamate as vital metabolites. Braz J Med Biol Res. 2003; 36:153-163.

[44] E. Jauniaux, J. Hempstock, C. Teng, et al. Polyol Concentrations in the Fluid Compartments of the Human Conceptus during the First Trimester of Pregnancy: Maintenance of Redox Potential in a Low Oxygen Environment. J ClinEndocrinolMetab. 2005; 90:1171-1175. 
562 [45] R.L. Phelps, B.E. Metzger, N. Freinkel. Carbohydrate metabolism in pregnancy. XVII. 563 Diurnal profiles of plasma glucose, insulin, free fatty acids, triglycerides, cholesterol, and 564 individual amino acids in late normal pregnancy. Am J ObstetGynecol. 1981; 140:730-736.

[46] P.M. Catalano, N.M. Roman-Drago, S.B. Amini, E.A.H. Sims. Longitudinal changes in body composition and energy balance in lean women with normal and abnormal glucose tolerance during pregnancy. Am J ObstetGynecol. 1998; 179:156-165.

[47] L.A. Barbour, C.E. McCurdy, T.L. Hernandez, et al. Cellular Mechanisms for Insulin Resistance in Normal Pregnancy and Gestational Diabetes. Diabetes Care. 2007; 30 (Suppl) S112-S119.

[48] M. Brownlee. Biochemistry and molecular cell biology of diabetic complications. Nature. $2001 ; 414: 813-20$.

[49] P. Haggarty. Effect of placental function on fatty acid requirements during pregnancy. Eur J ClinNutr. 2004; 58:1559-1570.

[50] W.W. Lam, J. Kirk, N. Manning, et al. Decreased cholesterol synthesis as a possible aetiological factor in malformations of trisomy 18. Eur J Med Genet. 2006;49:195-199.

[51] N. Gruchy, N. Bigot, C. Jeanne Pasquier, et al. Involvement and alteration of the Sonic Hedgehog pathway is associated with decreased cholesterol level in trisomy 18 and SLO amniocytes. Mol Genet Metab. 2014; 112:177-182. 
Table 1. Demographic and clinical features of patients with fetuses affected by chromosomal disorders and controls

\begin{tabular}{|c|c|c|c|c|c|}
\hline \multirow{2}{*}{} & \multicolumn{5}{|c|}{ Patients information } \\
\cline { 2 - 6 } & $\mathrm{N}$ & $\begin{array}{c}\text { Age (years) } \\
\text { (Average } \pm \\
\text { SD) }\end{array}$ & $\begin{array}{c}\text { NT (mm) } \\
\text { Average } \pm \\
\text { SD) }\end{array}$ & $\begin{array}{c}\text { CRL (mm) } \\
\text { (Average } \pm \text { SD) }\end{array}$ & BMI \\
\hline Euploids & 13 & $36.07 \pm 4.9$ & $2.12 \pm 1.67$ & $59.10 \pm 10.6$ & 19.6 \\
\hline Aneuploids & 8 & $35.75 \pm 6.2$ & $3.98 \pm 0.5$ & $60.31 \pm 9.7$ & 20.8 \\
\hline
\end{tabular}

Abbreviations. NT= Nuchal Translucency; CRL=Crown Rump Length, BMI= Body Mass Index. 
Table 2. Panel of the most discriminant metabolites resulting from the multivariate analysis. In the table were included the VIP value ( $>1$ for the metabolites assayed with ${ }^{1} \mathrm{H}-\mathrm{NMR}$ and GC-MS), the p-value (U-Mann Whitney test) with the respective correction for multiple comparisons (BenjaminiHochberg) for each metabolite. Metabolites resulting from the HPLC analysis did not show VIP values since they underwent only univariate analysis and not multivariate analysis.

\begin{tabular}{|c|c|c|c|c|}
\hline & Variables & VIP value & P-value & $\begin{array}{c}\text { Benjamini- } \\
\text { Hochberg }\end{array}$ \\
\hline \multirow{10}{*}{$\frac{\propto}{\Sigma}$} & Myo-Inositol & 1.44293 & 0.01 & 0.036 \\
\hline & Lactate & 1.18766 & 0.1 & - \\
\hline & Asparagine & 1.87244 & 0.3 & - \\
\hline & Glycerol & 1,00025 & 0.4 & - \\
\hline & Valine & 1,52049 & 0.8 & - \\
\hline & Leucine & 1,11058 & 0.02 & 0.036 \\
\hline & Isoleucine & 1,49307 & 0.02 & 0.036 \\
\hline & Fumarate & 1.05523 & 0.32 & - \\
\hline & Betaine & 1,09841 & 0.16 & - \\
\hline & Acetate & 1.02471 & 0.07 & - \\
\hline \multirow{11}{*}{ 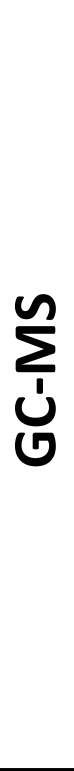 } & Pyruvate & 1,96127 & 0.2 & e \\
\hline & Homoserine & 1,02222 & 0.5 & - \\
\hline & Dehydroascorbic acid & 1,19049 & 0.03 & 0.038 \\
\hline & D-fructose & 1,04314 & 0.07 & - \\
\hline & 1,5-anydro-D-sorbitol & 1,08823 & 0.1 & - \\
\hline & Glucose & 1,22809 & 0.04 & 0.045 \\
\hline & d-Sorbitol & 1,18827 & 0.09 & - \\
\hline & Inositol & 1,22475 & 0.08 & - \\
\hline & Palmitic acid & 1,31942 & 0.02 & 0.036 \\
\hline & Stearic acid & 1,24499 & 0.05 & NS \\
\hline & Cholesterol & 1,57857 & 0.004 & 0.036 \\
\hline \multirow{3}{*}{$\begin{array}{l}\cup \\
\underline{\mathbf{a}} \\
\underline{\mathbf{I}}\end{array}$} & GSSH & - & 0.11 & - \\
\hline & GSH & - & 0.23 & - \\
\hline & Vit. C & - & 0.03 & 0.038 \\
\hline
\end{tabular}



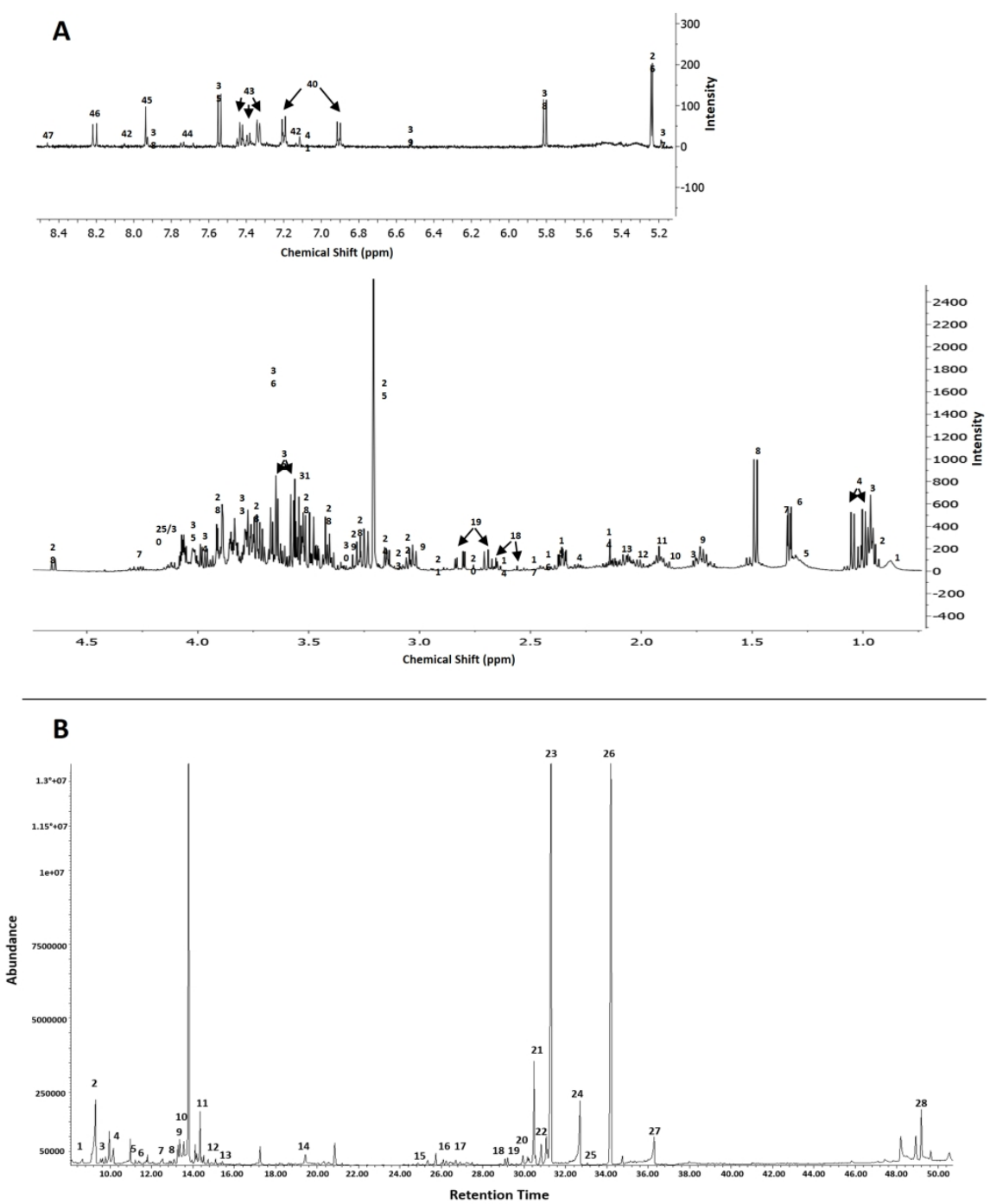

Figure 1. Identified compounds in chorionic villous samples analyzed with NMR and GC-MS.

Figure 1A. Identified compounds in NMR representative spectrum:1. Lipid1; 2. Isoleucine; 3. Leucine; 4. Valine; 5. Lipid 2; 6. Lactate; 7. Threonine; 8. Alanine; 9. Lysine; 10. Thymine; 11. Acetate; 12. Proline; 13. Glutamate; 14. Methionine; 15. Glutamate; 16. Pyroglutamate; 17 . Glutamine; 18 . Citrate; 19. aspartate; 20. Sarcosine; 21. Asparagine; 22. Creatine; 23; Ornithine; 24. Ethanolamine; 25. Choline; 26 O-phosphocholine; 27. Glycero-phosphocholine; 28. Glucose; 29. Betaine; 30. Myo-inositol; 31. Glycine; 32. Glycerol; 33. Glucitol; 34. Serine; 35. Fructose; 36. Nucleotides; 37. Mannose; 38. Uracil; 39. Fumarate; 40. Tyrosine; 41. Histidine; 42. n-Methylhistidine; 43. Phenylalanine; 44. Tryptophan; 45. Xanthine; 46. Hypoxanthine; 47. Formate.

B. Identified compounds in GC-MS representative chromatogram: 1. Pyruvic acid; 2. Lactic acid; 3. L-valine; 4. L-alanine; 5. Leucine; 6. L-isoleucine; 7. Urea; 8. Ethanolamine; 9. L-serine; 10. Glycerol; 11. Glycine; 12. Succinic acid; 13. Fumaric acid; 14. Malic acid; 15. Homoserine; 16. Xylitol; 17. Ribitol; 18. Dehydroacorbic acid; 19. 1,5-anydro-D-sorbitol; 20. D-fructose; 21. D-glucose; 22. D-sorbitol; 23. DMannitol; 24. Palmitic acid; 25. Inositol;, 26. Myo-inositol; 27. Stearic acid; 28. Cholesterol 

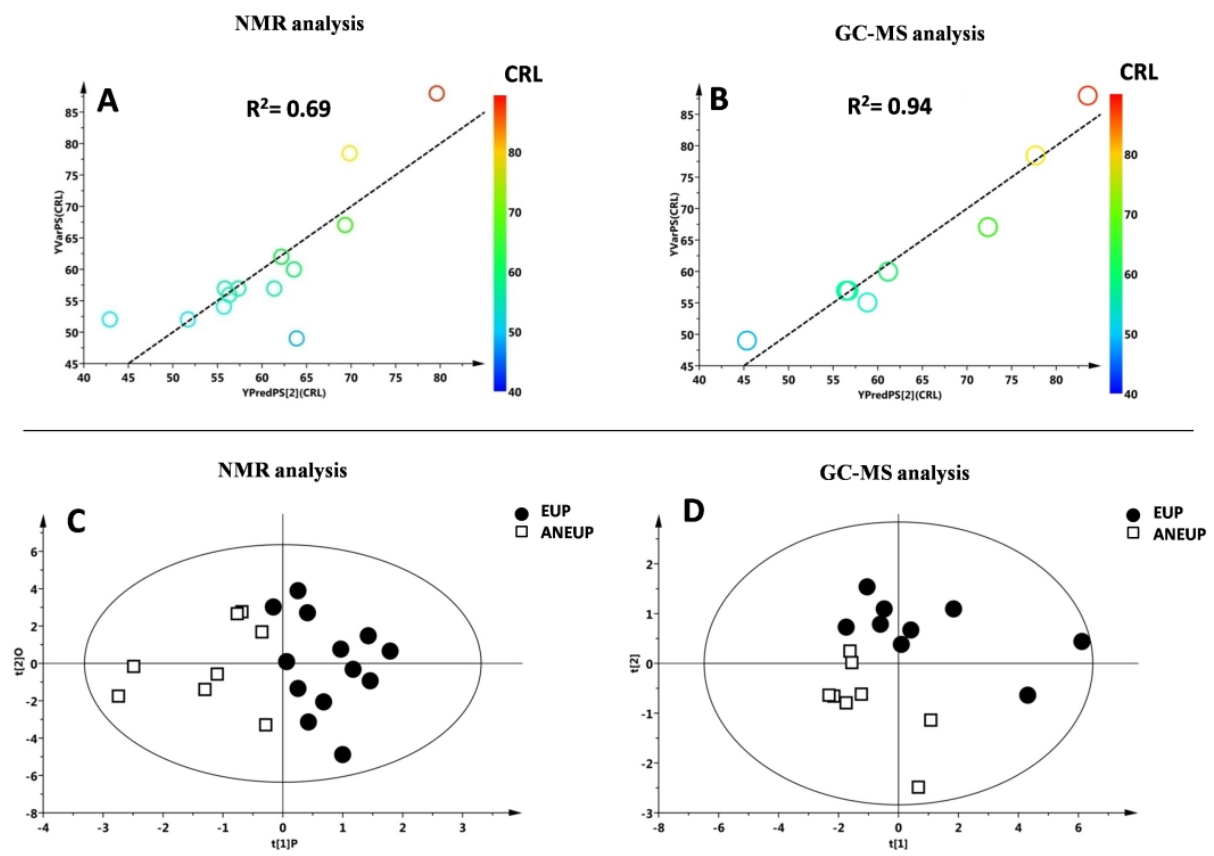

Figure 2. Partial least square regression analysis and partial least square discriminant analysis.

Figure 2. A-B PLS regression analysis. Correlation between the metabolic profile of chorionic villous and CRL parameter of fetuses (points represent control samples (group EUP) and the color indicated the respective

CRL value). $Y$ axis indicated the CRL value for each sample as reported by the clinical tests, while the predicitive CRL based on the metabolic profile is reported in the $X$ axis. A)The correlation analysis showed a $\mathrm{R} 2=0.69$ for the NMR analysis and B) $\mathrm{R} 2=0.94$ for the GC-MS analysis. C-D. PLS-DA models of samples with controls (black circles, group EUP) and chromosomal disease (white boxes, group ANEUP). For the NMR analysis (C) statistical parameters were $\mathrm{R} 2 \mathrm{X}=0.70, \mathrm{R} 2 \mathrm{Y}=0.65, \mathrm{Q} 2=0.30$, while for the GC-MS analysis (D) were $\mathrm{R} 2 \mathrm{X}=0.60, \mathrm{R} 2 \mathrm{Y}=0.75, \mathrm{Q} 2=0.47$. The models were then validated with the permutation test, (for the NMR R2 intercept $=0.428$; Q2 intercept $=-0.12$, for the GC-MS R2 intercept $=0.47$; Q2 intercept $=-0.6$ ). 


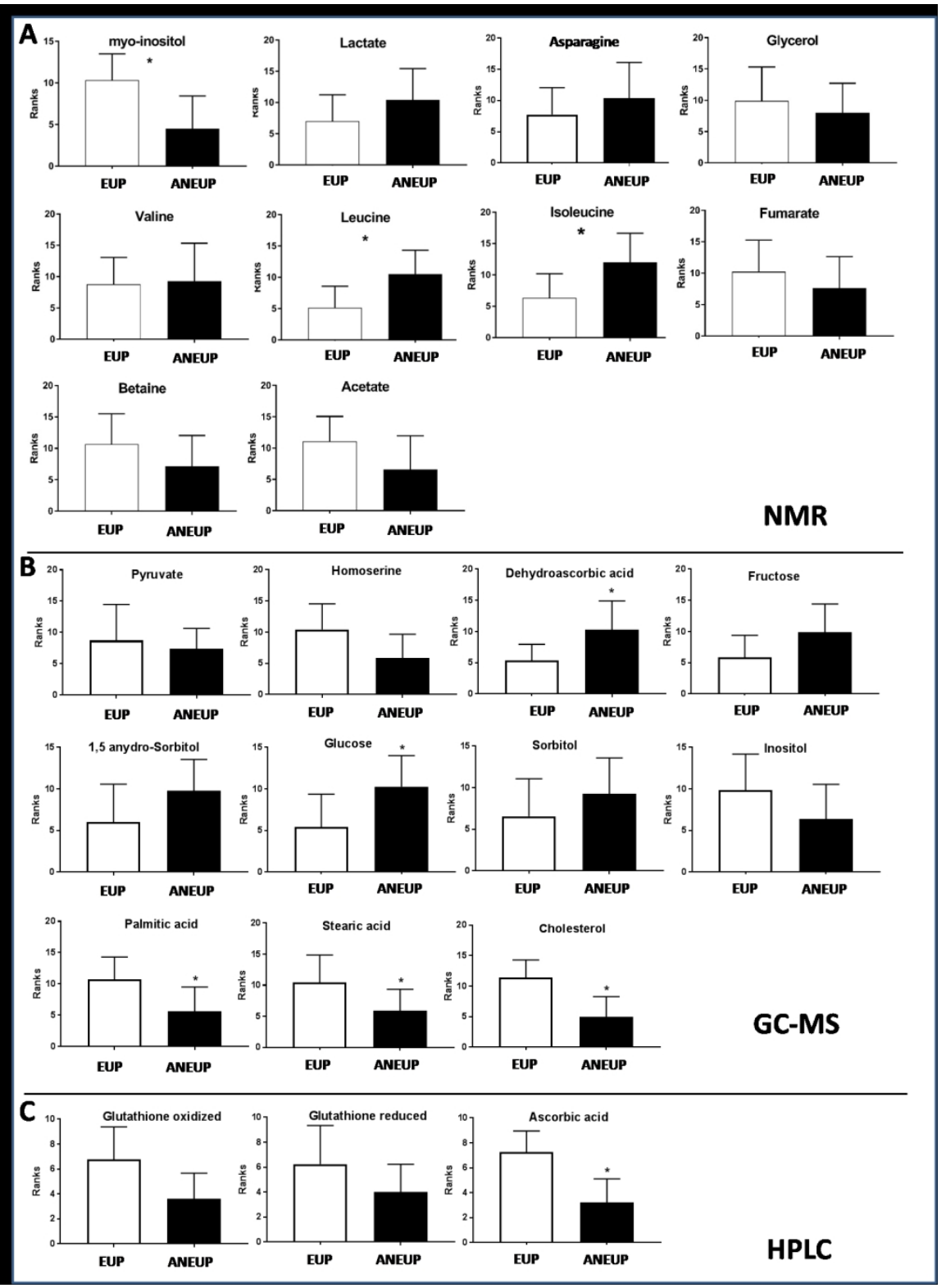

Figure 3. Comparison of select metabolites assayed in chorionic villous of fetuses with normal and abnormal karyotype.

Figure 3. The most important metabolites expressed by ranks with standard deviations as determined by different techniques. Group EUP were cases with normal chromosomes and group ANEUP were cases with abnormal chromosomes. Statistical analysis was performed by Mann-Whitney U test. Metabolites differences between groups with p-value $<0.05$ are indicated with the star. A) Analysis by NMR. B) Analysis by GC-MS C) Analysis by HPLC: box plots of the intracellular aminothiols such as GSH, GSSG and ascorbic acid. Data is expressed as area of peak to $\mu \mathrm{g}$ of proteins.

$$
258 \times 352 \mathrm{~mm}(150 \times 150 \mathrm{DPI})
$$



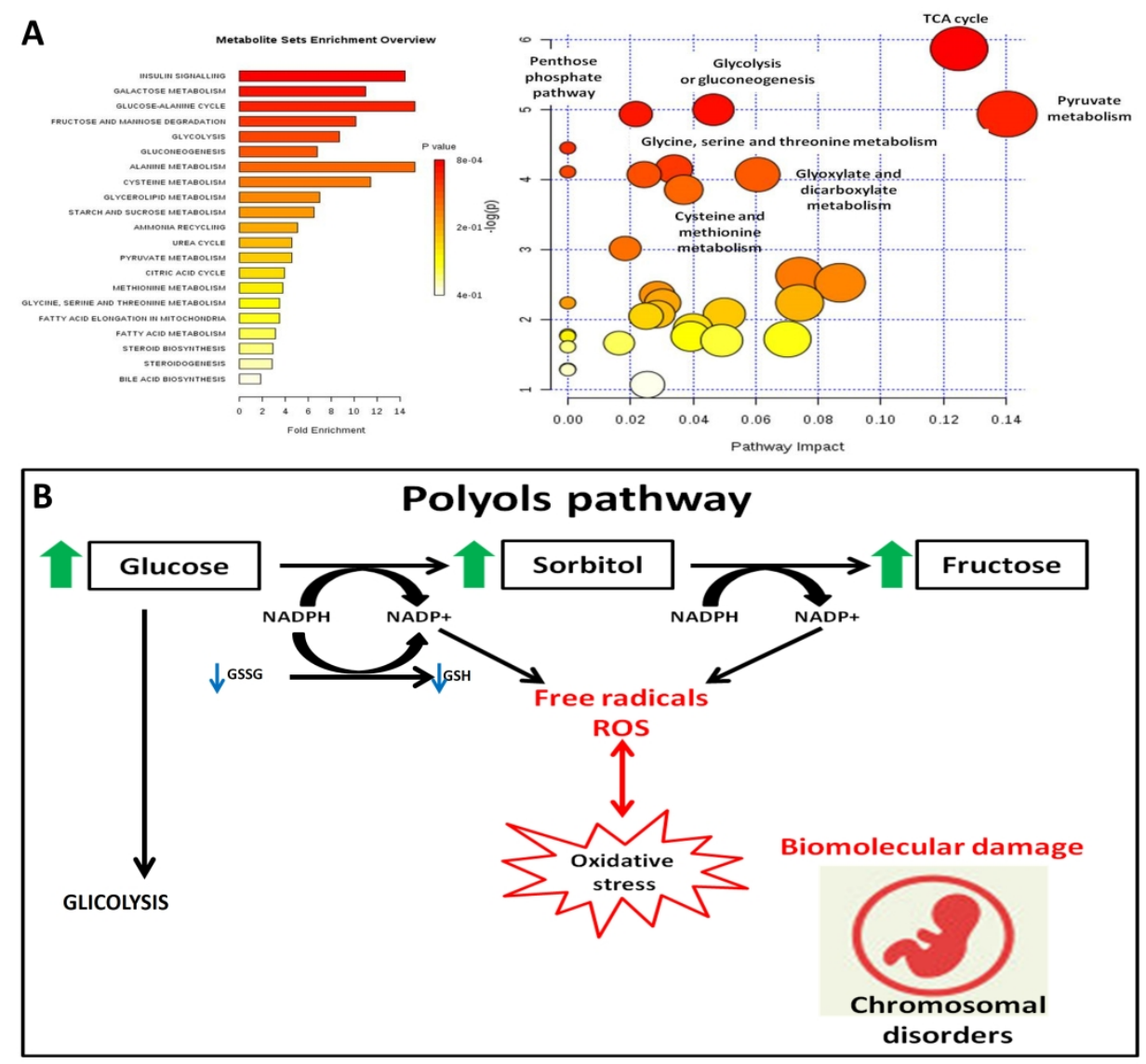

Figure 4. Pathways analysis.

Figure 4. Most important pathways involved in placentas in cases with abnormal chromosomes. A) Summary of the most important pathways resulting from the analysis with Metaboanalyst: TCA cycle, pyruvate metabolism, glycolysis and gluconeogenesis, pentose phosphate pathway, glycine, serine and threonine metabolism were the most involved nets. The colours and the size of the circles indicate the importance of the pathway, evaluated through statistical parameters such as match status (metabolites resulted discriminant in our analysis and involved in the pathway), p-value and FDR correction (all parameters were reported in the supplementary material) calculated by the software Metaboanalyst. The red colour and the big size of the circles indicate the most important pathways. B) Roles of the identified metabolites (green arrows means increase while blue arrows decrease) involved in the polyols pathway.

$267 \times 259 \mathrm{~mm}(300 \times 300$ DPI $)$ 

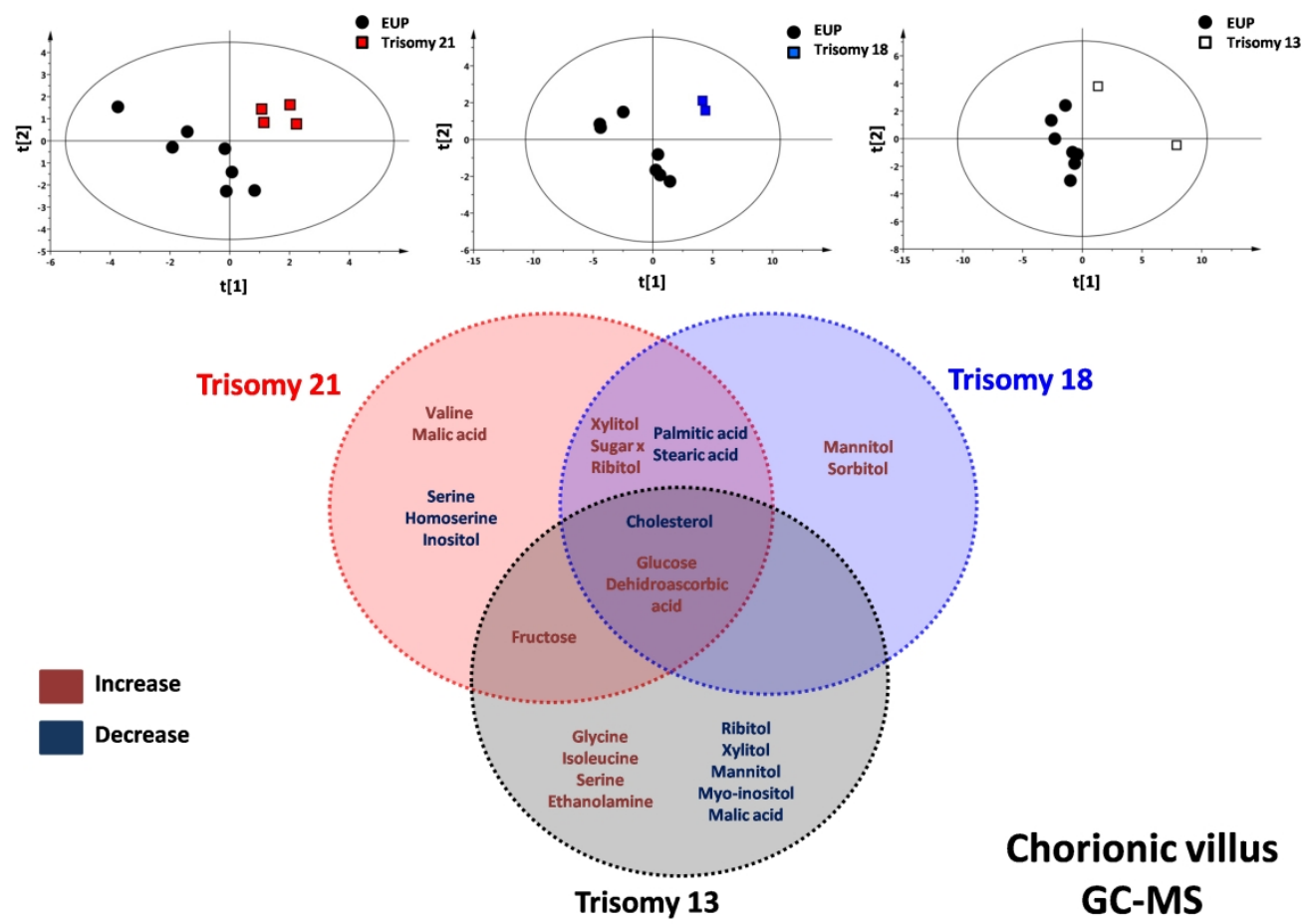

Figure 5. Partial least square discriminant analysis of trisomy 21 , trisomy 18 , trisomy 13 and control Group. Figure 5A. PLS-DA models of the three different chromosomal diseases $(21, n=4,18, n=2$ and $13, n=2)$ and control group built with the GC-MS data (C). Statistical parameters were group EUP vs Trisomy 21, $\mathrm{R} 2 \mathrm{X}=0.62, \mathrm{R} 2 \mathrm{Y}=0.887, \mathrm{Q} 2=0.441$, group EUP vs Trisomy 18: R2X=0.509, $\mathrm{R} 2 \mathrm{Y}=0.940, \mathrm{Q} 2=0.431$, group EUP vs Trisomy 13: R2X $=0.557, \mathrm{R} 2 \mathrm{Y}=0.868, \mathrm{Q} 2=0.399 \mathrm{~B}$. Venn diagram showing metabolite relationships between trisomy 21, trisomy 18 and trisomy 13.

$254 \times 190 \mathrm{~mm}(300 \times 300 \mathrm{DPI})$ 